\title{
How to Find Multiple Systems Underlying a Two-Way Table of 0's and 1's, With Applications to Cognitive Impairments and Medical Laboratory Science
}

\author{
T. P. Hutchinson \\ University of Adelaide
}

\begin{abstract}
Datasets are sometimes encountered that consist of a two-way table of 0's and 1's. For example, this might show which patients are impaired on which of a battery of tests, or which compounds are successful at inactivating which of several micro-organisms. The present paper describes a method of analysing such tables, that reveals and specifies two (or more) systems or modes of action, if indeed they are needed to explain the data. The approach is an extension of what, in the context of cognitive impairments, is termed double dissociation. In order to be simple enough to be practicable, the approach is deterministic rather than probabilistic.
\end{abstract}

Key words: Binary data, cognitive impairment, double dissociation, inactivation of micro-organisms, item response theory, micro-organisms' utilisation of different compounds, Rasch model.

\section{Introduction}

This paper describes a method of data analysis that may be useful in several areas of medical science. The starting point is a particular format of data, as follows.

- There is a two-way table of 0's and 1's.

- The 0's and 1's represent a dependent variable, that is, an outcome perhaps, failure and success, or death and life, or metabolised and not metabolised.

- The rows represent categories of one factor, or independent variable, and the columns those of a second. (Referring to an "independent variable" may seem to be peculiar language if, for example, the rows represent different people. The idea is to emphasise that the 0's and 1's are viewed as being determined by, or predictable from, knowledge of which row and which column we are referring to.) 
A method of summarising such data will be proposed that reveals two (or more) distinct systems involved in generating the pattern of 0's and 1's, if such is indeed the case. (There is no great significance in the use of the word system. Other words such as process or function or module or mode of action might be equally appropriate.)

I should straightaway alert the reader to an important limitation of the method. This is that it may take too much advantage of chance, and reveal two systems when really there is only one system plus random errors, or even the assumptions of the method bear no resemblance to scientific reality. In other words, there is no form of standard error available, and no test for comparing the fit of two systems with that of one system. However, there will often be other information available, concerning how the row categories differ one from another, and how the column categories differ one from another. As the method does not use this, it will provide an independent check on the plausibility of the different systems proposed. In any case, data exploration and hypothesis generation are valid activities, just as statistical testing is.

The next Section will sketch assumptions of the method, contrasting it with the Rasch model, which is a common way of approaching such a dataset. Sections 3 and 4 will show how data may be explored so as to reveal two or more processes, if indeed the data do require them. The examples are datasets on cognitive impairments. Section 5 applies the method to the inactivation of micro-organisms by different compounds (e.g., antibiotics), to the occurrence of various antigens in

cancer patients, and to the classification of micro-organisms according to which compounds they can metabolise. Section 6 suggests that it may be possible to adapt the method to datasets in which the dependent variable is not merely binary, but has more detail. Section 7 is discussion.

\section{Method, and Contrast with the Rasch Model}

Some readers may be familiar with the following approach to a table of 0's and 1's; this is described here only to serve as a contrast to the real topic of the present paper. The notation will be that the rows are labelled by $i$, and the columns are labelled by $j$.

- There exist quantities $R_{i}$, characteristic of the rows.

- There exist quantities $C_{j}$, characteristic of the columns.

- The logit of the probability of cell $(i, j)$ being 1 is $R_{i}-C_{j}$. (The logit of a probability $p$ is $\ln (p /(1-p))$, where $\ln$ means natural logarithm.)

This is termed the Rasch model. See, for example, Yen (1992). It is commonly used in such fields as ability testing. Rows represent people, columns represent 
items, and the outcome is whether or not the person succeeded in answering the item. Quantities $R_{i}$ and $C_{j}$ are estimated from the data, $R_{i}$ being interpreted as the ability of person $i$, and $C_{j}$ as the difficulty of item $j$. The topic, in which variations of the Rasch model are also used, is often known as item response theory.

Notice two features of the Rasch model. First, it imposes unidimensionality on the rows and on the columns, that is, the people are described by a single characteristic, and the items are described by a single characteristic. Second, it is a probabilistic model: it does not say that someone high in ability attempting an item low in difficulty will always be successful, or that someone low in ability attempting an item high in difficulty will never be successful, merely that the probabilities are high and low respectively. These features (unidimensional, probabilistic) are not in the model I will discuss. Instead, I will assume that two or more characteristics are needed to describe each person, and correspondingly two or more characteristics are needed to describe each item. The price to be paid for this greatly increased flexibility is that, in order to be simple enough to use, the model will be deterministic, not probabilistic.

Unidimensionality of the Rasch model means that when examining data from this viewpoint, it is impossible to discover any interesting structure to it, as that has all been assumed away by the model. Of course, I am referring to structure beyond single quantities for the rows and the columns: there are many contexts where a single quantity for each row (e.g., ability) and a single quantity for each column (e.g., difficulty) is exactly what is wanted.

The method to be proposed below may be expressed as follows. Let the two characteristics for person $i$ be $R_{i}$ and $S_{i}$, and the two characteristics for item $j$ be $C_{j}$ and $D_{j}$. The outcome of one process is hypothesised to be determined by whether the difference $R_{i}-C_{j}$ is positive or negative, and the outcome of a second process by whether the difference $S_{i}-D_{j}$ is positive or negative. The two outcomes are then combined into the observed outcome by some rule: this rule might be "the outcome is success if both processes are successful", or it might be "the outcome is success if either process is successful". Obviously, this could be extended to three or more person characteristics, and correspondingly three or more item characteristics. (Actually, the notation just presented, with one difference $R_{i}-C_{j}$ and another $S_{i}-D_{j}$, has been introduced to make clear the similarity with, and the difference from, the Rasch model. The notation is not needed in this paper: the outcome of the first process is implied by whether $R_{i}$ is larger or smaller than $C_{j}$, and calculations of the difference and then some function of this are not needed. The same goes for the second process.) To date, I have used this method in three contexts - cognitive impairments (Hutchinson, 2003), antimicrobial chemicals (Hutchinson, 2004), and antigens in cancer 
patients (Hutchinson, 2005).

In empirical studies, one typically wants to know (for example) which patients are impaired on which tasks, or which micro-organisms are inactivated by which chemicals, so the usual way of presenting the data is as the full table of 0's and 1 's. When interest focuses on patterns, the dataset that needs to be analysed is often appreciably smaller and simpler than appears when looking at the full table. Example 1 starts as 39 patients by 4 tests, but only eight distinct patterns of responses need to be considered. Example 3 starts as 33 compounds by 7 dermatophytes, but only eleven distinct patterns will need to be considered.

\section{Cognitive Impairments from Brain Injury or Disease: The Impor- tance of Double Dissociations}

The purpose of this Section is to give details of the model, with several artificially simple examples. The model has various applications, but here the approach is to use the specific language of cognitive impairment.

\subsection{People, tasks, and patterns of performance}

Patients with injuries or disease of the brain can provide many valuable clues to how the healthy brain works. The term "double dissociation" means that one patient can do Task A but not Task B, while a second patient can do Task B but not Task A. Double dissociations are important for inferring mental mechanisms. In the absence of information from the second patient, finding that Task A but not Task B can be performed (i.e., a single dissociation) could be put down to Task A being easier, which is fairly uninteresting. In the absence of information from Task B, finding that one patient but not another can perform Task A could be put down to the second patient being more severely damaged, which again is fairly uninteresting. Demonstrating different patterns of performance in different patients suggests that the tasks call upon different systems, one of which is damaged in the first patient and the other in the second patient. The suggestion is by no means a logical necessity: see Section 7 for discussion of how a nonmonotonic dependence of performance on some variable can lead to double dissociation.

Concerning double dissociation, see, for example a special issue of Cortex, issue 1 of volume 39 (2003). See Coltheart (2001) for an account of how evidence from patients with cognitive impairments is used in theory-building. There is, however, very little in the literature about the processing of data. The present paper might be seen as helping to fill that gap. The idea of different systems certainly does not contradict the concept of degrees of difficulty of tasks, and degrees of impairment of patients. What will be proposed is that when there is data on several tasks and several patients, we try to find two systems (or perhaps 
three) that will account for the double dissociations in the data, with each system consisting of a list of tasks, ordered by difficulty, that implies single dissociations.

A homely example might be constructed around school subjects.

- Suppose that for successful performance of a task in writing (W), successful performance of a task in grammar $(\mathrm{G})$ is necessary but not sufficient. Then the following patterns of failure might be observed: W, WG. (That is, G alone is not observed, as failure in $\mathrm{G}$ implies failure in $\mathrm{W}$ also.)

- Suppose that for successful performance of a task in calculus (C), successful performance of a task in elementary linear algebra $(\mathrm{L})$ is necessary but not sufficient, and (further) that for successful performance of the elementary linear algebra task, successful performance of an arithmetic task (A) is necessary but not sufficient. Then the following patterns of failure might be observed: C, CL, CLA. (That is, A alone, L alone, AL, and AC are not observed.)

- The language and the mathematics tasks might be quite distinct, with all of the following additional patterns of failure being observed: WC, WCL, WCLA, WGC, WGCL, WGCLA.

Some other task might call upon both the language and the mathematics systems. For example, successful performance of a task in statistics might require successful performance of both $\mathrm{G}$ and $\mathrm{A}$.

\subsection{Inactivation of micro-organisms}

As a foretaste of other applications (see Section 5), I might mention that in medicinal chemistry, there is much interest in screening a variety of compounds for success in inactivating micro-organisms, and datasets are published showing which inactivate which. A possibly fruitful way of handling such data is to follow the double dissociation reasoning. If a compound inactivates one organism but not another, we are not surprised, but simply say that the first organism is less tolerant (or more sensitive) than the second. If an organism is inactivated by one compound but not by another, we are not surprised, but simply say that the first compound is in some sense stronger than the second. But if we see that some compounds inactivate $\mathrm{A}$ but not $\mathrm{B}$, whereas others inactivate $\mathrm{B}$ but not $\mathrm{A}$, this cannot be explained by the compounds differing in strength, or by the organisms differing in tolerance. It might be considered evidence there are at least two systems in the organisms that may be vulnerable to this set of compounds (that is, two modes of action of these compounds on these organisms). 


\subsection{Two tasks}

Double dissociation implies that our dataset consists of a patient-by-task matrix, with the entries in the matrix being 0 or 1 to indicate that the patient failed or succeeded in the task. (That is, the outcome is binary - there is no partial success.) The simplest example of double dissociation is two patients attempting two tasks, with each succeeding in the task that the other fails. If we call the two tasks $\mathrm{A}$ and $\mathrm{B}$, the patient-by-task matrix is:

$\begin{array}{lr}\text { One patient } & A B \\ \text { Another patient } & 01 \\ & 01 \\ (0=\text { failure, } 1=\text { success }) .\end{array}$

\subsection{Three tasks (1)}

Now suppose there are three tasks, and the only patterns observed in a number of patients are:

$\begin{array}{ll} & \text { ABC } \\ \text { Patient 1 } & 101 \\ \text { Patient 2 } & 011 \\ \text { Patient 3 } & 100\end{array}$

That is, the list of patterns of tasks that are failed consists of B, A, BC. Notice that $\mathrm{A}$ and $\mathrm{B}$ may alone be failed, and $\mathrm{C}$ is only failed when $\mathrm{B}$ also is failed. On the logic of double dissociation, $\mathrm{A}$ and $\mathrm{B}$ must be performed by different systems. When attempting to find systems each consisting of a list of tasks ordered by difficulty, what we get without considering $\mathrm{C}$ is:

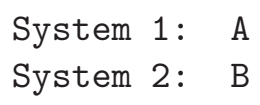

The two lines are (the beginnings of) lists: A is the most difficult task for System 1 , and B is the most difficult task for System 2. As they are the most difficult, either may be failed without any other task being failed. Turning now to $\mathrm{C}$, the list of patterns of failed tasks includes BC as well as B, but it does not include C alone. This is interpreted as $\mathrm{C}$ being performed by the same system as $\mathrm{B}$, with $\mathrm{C}$ being easier. This is represented as follows:

System 1: A

System 2: B C 
Without further information, each system is no more than an ordered list of tasks. There may or may not be some other source of information (concerning the patients, or the tasks) that permits plausible speculation about the nature of the systems, and there may or may not be reason for choosing some word other than system (such as function or module). See Section 3.8.

The patients can be positioned within the systems. Let the patients be labelled 1, 2, 3, as shown above, and consider patient 1, who was impaired on task B only. Is there a pair of positions, within the two systems above, that implies impairment on B and no other task? Yes, the only task to the left of 1 in either list below is B:

System 1: 1 A

System 2: B 1 C

Similarly, patient 2, who was impaired on A only, is in these positions:

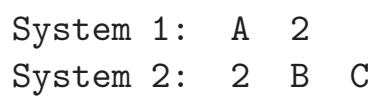

And patient 3, who was impaired on $\mathrm{B}$ and $\mathrm{C}$, is in these positions:

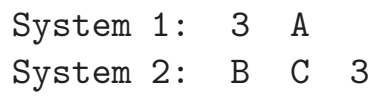

For this (small, hypothetical) dataset, the patterns of impairment can be reproduced without error. In summary, we could write

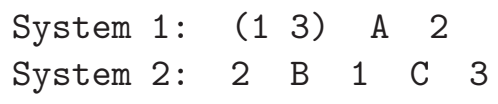

(In System 1, patients 1 and 3 are bracketed together to indicate we cannot distinguish their relative order. Obviously, if there were patients showing identical impairment patterns, they could not be distinguished, either.) In this set of patients, none have damage to both systems. Damage to both systems would lead to impairment on both A and B.

The right hand ends of the lists that make up the systems are not fully specified: it is possible, though not necessary in order to account for this dataset, that sufficiently severe damage to System 2 could result in task A being failed, as well as B and $\mathrm{C}$, and that sufficiently severe damage to System 1 could result in $\mathrm{B}$ and/or $\mathrm{C}$ being failed, as well as $\mathrm{A}$.

\subsection{Three tasks (2)}

Now suppose instead that the patterns observed are as follows: 


$\begin{array}{ll} & \text { ABC } \\ \text { Patient 1 } & 011 \\ \text { Patient 2 } & 010 \\ \text { Patient 3 } & 000\end{array}$

This contrasts with the dataset of Section 3.4, as it can be explained by postulating only one system:

\section{System 1: A C B}

Light damage to this system leads to task A (only) being failed (as in patient 1), more severe damage leads to both $\mathrm{A}$ and $\mathrm{C}$ being failed (as in patient 2), and sufficiently severe damage leads to all three tasks being failed (as in patient 3).

System 1: A 1 C

\subsection{Consider patterns of non-impairment, also}

Now suppose that the patterns of impairment observed are:

$\begin{array}{ll} & \text { ABC } \\ \text { Patient 1 } & 011 \\ \text { Patient 2 } & 101 \\ \text { Patient 3 } & 110 \\ \text { Patient 4 } & 001 \\ \text { Patient 5 } & 010\end{array}$

Here, each of the tasks is the only one failed in one or other of the patients: only $\mathrm{A}$ in patient 1 , only $\mathrm{B}$ in patient 2 , and only $\mathrm{C}$ in patient 3 . A model of the type discussed so far will require three systems, and cannot be any simpler than a list of the tasks themselves. But interchange 0 and 1 and apply the same method of reasoning. That is, switch attention to the patterns of tasks that are not impaired. These are (in order from the bottom of the table to the top) B, $\mathrm{C}, \mathrm{AB}, \mathrm{AC}, \mathrm{BC}$. This list of combinations can be transformed into two systems in the way that is now familiar. B and $\mathrm{C}$ occur on their own, and so are in the leftmost positions:

System 1: B

System 2: C

Pattern AB implies:

System 1: B A

System 2: C 
Pattern AC implies:

System 1: B A

System 2: C A

Pattern BC is compatible with these two systems, and thus they are a complete description of the data. Here, non-impairment of a task on a system occurs if the task is to the left of the patient, and non-impairment of a patient on a task occurs if the task is to the left of the patient on either system. Four patterns are the result of some non-impairment in one system only, and $\mathrm{BC}$ implies some non-impairment of both systems.

\subsection{Equivalence of two types of model}

In Sections 3.4 and 3.5, it was supposed that the tasks that are impaired are those to its left in either system. The idea is that both systems are essential to performing the task. Equally reasonable is the idea that the systems are alternative ways of performing a task, and thus that impaired tasks must be to the left in both systems. The easiest way of obtaining a model of this type may be to proceed as in Section 3.6: turn attention to patterns of non-impaired tasks. A model is found in which tasks are unimpaired if they are unimpaired in either system. But to say this is the same as saying tasks are impaired if they are impaired in both systems.

\subsection{What do we know about the systems?}

The model that is obtained consists of two (or more) ordered lists of tasks, along with the positions of the patients in those lists. To say anything further about the systems is to go beyond the data. Very often, we will want to go beyond the data and speculate about the systems. We might say that system 1 is for recognising letters, whereas system 2 is for remembering words, for example. Or, if the data is about which compounds kill which bacteria, one system might refer to penetration of the cell wall and the other to doing the damage inside.

The model is determined by the data. Thus if any interesting interpretations are found (compatibilities with other datasets or some theory, or correspondence with ideas about what the tasks are tapping or what pathologies the patients have), these will have their full value, as they have not been part of the input to the model. Nevertheless, sometimes a more active approach to the data analysis might be preferred, in which some preconceptions or theoretical ideas are imposed. In this case, the preconceptions would need to be expressed as an allocation of tests (for example) to systems, together with ordering of difficulty of 
tests within systems. The data analysis would then take the preconceptions as the starting point, and add greater detail to them.

\section{Two Datasets on Cognitive Impairments}

\subsection{Example 1}

Papagno (2001) listed the impairments of 39 patients with Alzheimer's disease on a set of four tests. These tests will be labelled T (token test), F (fluency), $\mathrm{M}$ (metaphors), and I (idioms). The observed patterns of impairments and the respective numbers of patients may be summarised as follows:

$\begin{array}{ll}\text { TFMI } & \\ 1111 & (10 \text { patients) } \\ 0111 & (3) \\ 1011 & (3) \\ 0011 & (9) \\ 0101 & (1) \\ 0110 & (3) \\ 1100 & (1) \\ 0010 & (1) \\ 1000 & (3) \\ 0000 & (5)\end{array}$

The data are from Table 1 of Papagno (2001); Papagno's codes 0 and 1 have been interpreted as impairment and 2, 3, 4 as normal performance.

Papagno gave a conventional (narrative) account of results and their implications, including such issues as whether double dissociation occurred between tests of propositional and of figurative language. Some features of that account come over clearly, but concerning the patterns of impairment, Papagno's discussion is complex and difficult to follow. This is a characteristic of multi-task multi-patient research in general, not a criticism of Papagno's paper specifically. In a typical paper, the description of the results is lengthy and complicated, comments on other authors' results and expectations from theories are included, and in some papers there is discussion of individual patients' lesions. The format of summarising test results that has been proposed in Section 3 above shows clearly the implications of the results for the structure of cognitive processing: this will at least help authors communicate their message more clearly, and at best will constitute a credible model of cognitive structure. 


\subsection{From data to model}

Starting from the list of patterns of impairment observed by Papagno, a model is built up as described below. Systems, each consisting of a list of tests ordered from most damage-sensitive (or difficult) to least damage-sensitive (or easiest), are proposed. It is presumed that if a system is damaged so as to impair performance on a test, any other test that is more difficult for that system is also impaired. It is also presumed that both systems are essential, in the sense that damage to either will impair performance on the relevant test(s). There is no intention to imply anything about the ordering of the two systems.

As a preliminary, patients who are either unimpaired on all tasks or impaired on all tasks (15 patients in this dataset) can be omitted from consideration. They are not relevant to a discussion of patterns of impairment - low severity of damage is sufficient to explain no impairment, and high severity of damage is sufficient to explain complete impairment. These being omitted, the remaining observed patterns of impairment were T, F, TF, TM, TI, MI, TFI, and FMI.

Step 1. There are only two single-test impairments, T and F. This implies:

$$
\begin{array}{ll}
\text { System 1: } & \text { T } \\
\text { System 2: } & \text { F }
\end{array}
$$

(The number of systems must be at least as many as the number of different single-test impairments.)

Step 2. Among the two-tests-impaired patterns, TF is the result of both systems being impaired. As both TM and TI are observed, a three-system model is needed. One possibility is:

$\begin{array}{ll}\text { System 1: } & \text { T I } \\ \text { System 2: } & \text { F } \\ \text { System 3: } & \text { M }\end{array}$

Step 3. That MI is another pattern of impairments implies:

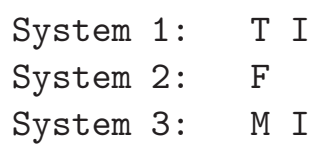

Step 4. And both the remaining patterns, TFI and FMI, are consistent with this, without further allocation of tests to systems.

Data from the re-testing of 23 patients some months later is given in Papagno's Table 4. There are two new patterns of impairment, FM and TMI. These are consistent with the model. 
Actually, the above is not quite the only possibility. The set of three systems, $\mathrm{T} \mathrm{M}, \mathrm{F} \mathrm{M}$, and I M is also consistent with the patterns observed at original test or re-test.

Further discussion of these results at this point would interrupt the presentation of Examples, and so is postponed to Section 7. Section 4.4 will give another example from the field of brain injury, and then examples from laboratory medicine will be presented.

\subsection{Patterns of non-impairment}

In Section 4.2, a three-system model was obtained. Might a two-system model be sufficient when starting from the list of patterns of non-impairment, as suggested in Section 3.6? No, a three-system model is required in this case also.

\subsection{Example 2}

Kemmerer et al. (2001) reported a dataset on the processing of verbs. There were 30 patients and 6 tests. The dataset was not compatible with a two-system model, and not even quite compatible with a three-system model (Hutchinson, 2003). A computer-aided analysis was used to obtain the best-fitting three-system model. The computing method was unsophisticated: there was no progressive homing-in on the best model, but rather the computer was used to generate all possible models consisting of two ordered lists (and then three ordered lists), to deduce the patterns of impairment predicted by each model, to count the patterns that were observed but not predicted, and to identify which model had fewest failures in its predictions. Thus the best model having three systems (all essential) was obtained. Of the 20 observed patterns of impairment, only one was inconsistent with this model. Interestingly, this model plainly resembled the characteristics of the tests that Kemmerer et al. identified before ever seeing the patterns of impairment. This dataset, with 6 tests, seemed to be at about the limit of what could be handled straightforwardly: there are 720 possible orderings of test difficulties, and therefore about 62 million different models made up of three systems (all essential).

When three or more systems are considered plausible, "all systems are necessary" and "any system is sufficient" are not the only possibilities. Others are: (i) two systems necessary, one of them consisting of two subsystems, either of which is sufficient, and (ii) either of two systems is sufficient, one consisting of two subsystems, both of which are necessary. In the course of examining the dataset of Kemmerer et al., consideration was given to such models, but none was an improvement on the best "three systems, all essential" model. 


\section{Further Examples of Areas of Application}

In this Section, five examples will be described that might be described as coming broadly within the field of laboratory medicine. Examples 3 and 5, like Example 2 above, have already been published: see Hutchinson $(2004,2005)$ for further details beyond those given here.

Perhaps the most common context for the format of data to be considered here is that of the effectiveness of a number of compounds against a number of pathogenic micro-organisms. Part of the interest in such data lies in each compound and each micro-organism, just as part of the interest in cognitive impairment data lies in each patient. But the total table of data is of interest, also. When examining such tables, several features are noticed successively:

- The average or typical level of effectiveness of this class of compounds against this list of micro-organisms;

- Which compounds are most effective and which are least effective, and which micro-organisms are most tolerant and which are least tolerant; and

- Whether some compounds are especially effective against one set of microorganisms, and other compounds against a different set of micro-organisms.

The second of these suggests an ordering of compounds and an ordering of microorganisms. The last of these suggests more than one ordering of compounds, and more than one ordering of micro-organisms, will be needed, and is the subject of the present paper.

\subsection{Example 3: Which micro-organisms are inactivated by which com- pounds}

Hutchinson (2004) applied the method described in Section 3 to data on the fungistatic activity (against 7 dermatophytes) of 33 compounds (2,4-dihydroxythiobenzanilides) (Matysiak et al., 2000). The 7 dermatophytes will be referred to by letters A to G. In the dataset of Matysiak et al., only the following response patterns of dermatophytes being inactivated were observed: None, B, E, AB, ABD, ABDE, ABEF, BEFG, ABEF, BEFG, ABDEF, ABDEFG, All. Obtaining a two-system theory proceeds as follows.

Step 1. In the case of at least one compound, only B is inactivated, and in the case of at least one other, only E. Therefore at least two systems are required, B being most sensitive for one and $\mathrm{E}$ being most sensitive for the other.

Step 2. AB was the only pattern having exactly two dermatophytes inactivated. This suggests that the theory so far should be: 


$\begin{array}{ll}\text { System 1: } & \text { B A } \\ \text { System 2: } & \text { E }\end{array}$

For example, if the second system is not damaged, first B alone and then both A and $\mathrm{B}$ are inactivated by damage to the first system.

Step 3. Consider patterns having exactly three dermatophytes inactivated. ABD was the only pattern of this kind observed, suggesting that the theory so far should be:

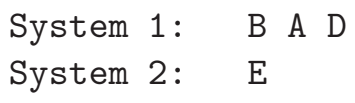

(Actually, at this point, the second system might be E B or even E B D, E A B D, etc. Most of these possibilities, but not E B, are eliminated at the next step.)

Step 4. Three patterns having exactly four dermatophytes inactivated were observed. ABDE requires no further elaboration of the theory, being predicted by what we already have. ABEF requires $\mathrm{F}$ to be added after $\mathrm{E}$; and $\mathrm{BEFG}$ requires $\mathrm{G}$ to be added after $\mathrm{F}$. Thus the theory so far is:

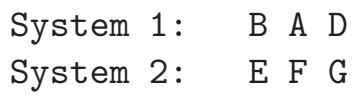

Step 5. Two patterns having exactly five or exactly six dermatophytes inactivated were observed, $\mathrm{ABDEF}$ and $\mathrm{ABDEFG}$. They require no further elaboration of the theory, as they are consistent with what we already have.

According to this theory, increasing strength of attack on the first system will lead to inactivation of first B, then successively A and D become inactivated also; increasing strength of attack on the second system will lead to inactivation of first $\mathrm{E}$, then successively $\mathrm{F}$ and $\mathrm{G}$ also; attack on both systems will lead to such patterns as inactivation of $\mathrm{B}$ and $\mathrm{E}$, of $\mathrm{B}$ and $\mathrm{E}$ and $\mathrm{F}$, and so on. Patterns such as inactivation of $\mathrm{A}$ alone, or inactivation of $\mathrm{D}$ and $\mathrm{F}$ and no others, are predicted never to be observed: if a compound is found that gives rise to such a pattern, it must be attacking some different system.

In the dataset of Matysiak et al. (2000), there are many compounds (33 of them) and few micro-organisms (only 7). It was convenient to express the systems as two ordered lists of micro-organisms. But positions of the different compounds are implied, and the systems could be expressed as two ordered lists of compounds (see Hutchinson, 2004). 


\subsection{Example 4: A second example of inactivation of micro-organisms}

In this example, there are few compounds and many micro-organisms. Table 2 of Beltrametti et al. (2003) lists which of 31 micro-organisms were inhibited by each of 4 antibiotics. There being so few antibiotics, let us express the systems in terms of these, rather than in terms of the micro-organisms. Let $\mathrm{A}$ to $\mathrm{D}$ be the antibiotics (A40926, DDC, MDC2, vancomycin, in each case at a concentration of $0.5 \mathrm{~g} / \mathrm{ml})$.

As with the cognitive impairments examples, we can imagine two types of theories.

- Inhibition of either system is sufficient to inhibit the micro-organism.

- Failure of inhibition via either system is sufficient for failure of inhibition of the micro-organism. This is equivalent to inhibition via both systems being necessary for inhibition of the micro-organism.

To consider the first of these, we start from the list of observed patterns of inhibition; we find that no two-system theory explains the data. So, then, consider the second possibility, by starting from the list of patterns of failure to inhibit. These patterns, listed with reference numbers, were:

$\begin{array}{ll}0 & \text { none } \\ 1 & \mathrm{D} \\ 2 & \mathrm{AD} \\ 3 & \mathrm{BC} \\ 4 & \mathrm{CD} \\ 5 & \mathrm{ABD} \\ 6 & \mathrm{BCD}\end{array}$

(That is, 1 refers to those micro-organisms for which only antibiotic D failed to inhibit it, 2 refers to those micro-organisms for which antibiotic A and antibiotic D both failed to inhibit it, and so on.) Proceeding in a similar manner to the previous example, the two-system theory shown below is obtained:

$$
\begin{array}{ll}
\text { System 1: } & \text { D A B } \\
\text { System 2: } & \text { C B }
\end{array}
$$

If the numbers identifying the micro-organisms are shown also, we get the following:

$$
\begin{array}{llllllllllll}
\text { System 1: } & \left(\begin{array}{llllllll}
0 & 3
\end{array}\right) & \text { D } & \left(\begin{array}{lllllll}
1 & 4 & 6
\end{array}\right) & \text { A } & \text { B } & 5 \\
\text { System 2: } & \left(\begin{array}{llllllll}
0 & 1 & 2 & 5
\end{array}\right) & \text { C } & 4 & \text { B } & \left(\begin{array}{llll}
3 & 6
\end{array}\right) &
\end{array}
$$


Reminder of interpretation: if an antibiotic (letter) is to the left of a microorganism (number), the system is not inhibited; if either system is not inhibited, the micro-organism is not inhibited. (As earlier, brackets indicate that order cannot be distinguished.)

\subsection{Example 5: Antigens in cancer patients}

Let A to E represent various MAGE (melanoma antigen) markers. The combinations giving positive results that were found in 37 lung cancer patients were as follows:

$\mathrm{A}, \mathrm{B}, \mathrm{C}, \mathrm{D}, \mathrm{E}, \mathrm{BC}, \mathrm{AD}, \mathrm{ABC}, \mathrm{ABE}, \mathrm{ACD}, \mathrm{ABCD}$

(Tables 1 and 2 of Mecklenburg et al., 2003: see Hutchinson, 2005). There seems to be a lot of variability, as any of the five markers might be the sole positive one. But let us list the data as combinations of negative results:

E, BE, DE, CD, ADE, BCE, ABCD, ABCE, ABDE, BCDE, ACDE

A simple form of dependence between the markers is a "negative implies negative" rule: a single ordered list such that if there is a negative result for any marker, there is also a negative result for all markers to its left. Such a list cannot be constructed for this dataset. But suppose there are two ordered lists of the markers, that each patient has a position in both lists, and that markers to the left of the patient in either list have negative results:

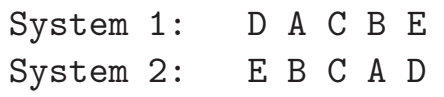

The above pair of lists generates all of the observed patterns except that one patient has negative results for $\mathrm{C}$ and $\mathrm{D}$. If we are bold, we might consider the pair of lists to be a theory, and interpret them as resiliencies of the different MAGE markers, with a patient's positions reflecting the strengths of two systems for suppressing them.

\subsection{Example 6: Which micro-organisms can utilise which compounds}

Table 1 of Singh et al. (2003) shows which of 20 compounds are utilised by which of 6 Pseudomonas strains. Let A to $\mathrm{F}$ represent the 6 strains. The dataset starts as a 20 by 6 table, but can be simplified considerably by omitting compounds utilised by all strains or by no strains, and omitting strains that were able to utilise all compounds or no compounds. (This results in $\mathrm{B}$ and $\mathrm{F}$ being omitted.) For one compound or another, the following combinations of strains were able to metabolise them: D, CDE, ACD, ACE. There are several possible pairs of systems that are consistent with this set of combinations. One of these is: 


\section{System 1: D E}

System 2: C A E

\subsection{Example 7: A second example of micro-organisms' utilisation of different compounds}

Table 2 of Vastag et al. (1998) shows which of 48 compounds could be utilised as the sole carbon source by which of 5 zygomycetous strains. Let A to E represent the 5 strains. For one compound or another, the following patterns of strains shown below did not show growth. (In preparing this list, slight growth has been counted as growth, ambiguous slight growth has been counted as no growth.)

$\begin{array}{rl}0 & \text { none } \\ 1 & \mathrm{C} \\ 2 & \mathrm{AE} \\ 3 & \mathrm{CE} \\ 4 & \mathrm{ABC} \\ 5 & \mathrm{ABE} \\ 6 & \mathrm{ACE} \\ 7 & \mathrm{CDE} \\ 8 & \mathrm{ABCD} \\ 9 & \mathrm{ABCE} \\ 10 & \mathrm{ACDE} \\ 11 & \mathrm{BCDE}\end{array}$

(That is, 1 refers to a compound for which $\mathrm{C}$ was the only strain not able to grow with it as the sole carbon source, 2 refers to a compound for which $\mathrm{A}$ and $\mathrm{E}$ were not able to grow, and so on.) Consider the following pair of systems:

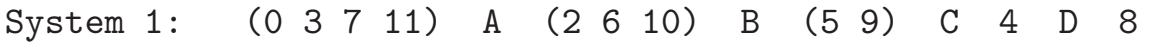

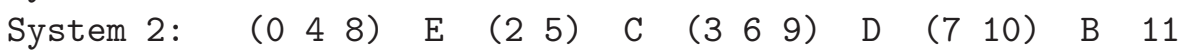

(Reminder of interpretation: if a strain (letter) is to the left of a compound (number), the system is not able to use the compound as the sole carbon source; if either system is not able to do this, the strain is not able to do this.) With the exception of one pattern $(\mathrm{C})$, this pair is consistent with all of the other patterns observed. The numbers identifying the compounds are shown as well as the letters representing the strains; brackets indicate that order cannot be distinguished; compound 9 can have another pair of positions besides that shown. 


\section{Processing of a More Precise Dependent Variable}

The method of data analysis described in this paper is designed for binary data, that is, 0's or 1's. Suggestions will now be made for adapting the method to more detailed data, as when a score on a test is available, or a grade describing vigour of growth.

Firstly, it may be possible to dismiss the detail in the response as being outside the area of interest. That is, the true response is viewed as genuinely binary, and the apparent detail arises through some sort of error. For example, as to cognitive impairments, a patient who is impaired might achieve partial performance by lucky guessing, or a patient who is unimpaired might partially fail because of a deficit in some system (e.g., motivation) outside the scope of the investigation. Or a laboratory procedure purporting to measure degree of activity might be well-known to be only reproducible to within plus or minus one grade. For any specific dataset, the likely error mechanism might guide the treatment of response detail when deriving the systems from the dataset. For example, if a patient has a motivation problem, underperformance in two tasks might be almost as likely as in one. Or, if the borderline between grades of activity is inherently difficult to judge, misreporting by two grades might be thought much less likely than two errors of one grade.

Secondly, what if we take seriously a response that is more specific than merely 0 or 1 ?

- Suppose the response is nominal (categorical). In such a case, it may be possible to identify two or more dimensions on which the responses differ, with these dimensions corresponding to different tasks. For example, if a patient spelled out the stimulus SHELL as S-H-E-E-L, this would be regarded as correct regarding the letters, correct regarding their order, incorrect regarding their numbers, and incorrect regarding whether or not it is an English word. Thus in place of the qualitative response of SHEEL being recorded for the single task of spelling, the set of four binary responses 1100 could be recorded. Similarly, if the qualitative response were S-ME-L-L, the set of four binary responses would be 0111. (McCloskey, 2003, mentions patients who made distinctive errors in attempting to spell words with double letters.)

- A more common starting point than a nominal classification of performance is a set of ordinal grades, or even numerical measurements. The straightforward way of proceeding is to choose some threshold for what constitutes

impairment, or in another context inhibition, and condense the grades into a binary classification, as in the pre-processing of data for Example 1. A 
more elaborate procedure would be as follows. Three grades is the simplest case. A task with three grades of performance might be treated as two tasks, one corresponding to achieving the highest grade, and one to achieving either of the two higher grades. (Suppose $1=$ low performance, $2=$ moderate, $3=$ high. Then instead of grade 2 being recorded for a single task, the set of two binary responses 01 would be recorded - i.e., not achieving the highest grade, but achieving the moderate grade. And in place of grades 1 and 3, 00 and 11 would respectively be recorded.)

These suggestions lead to replacing a table of a fine-grained dependent variable with a larger table of 0 's and 1's. A large table might require computerised analysis.

\section{Discussion}

The first part of the Discussion will use language specific to cognitive impairments. It could have been placed after Section 4.3, except that there it would have interrupted the presentation of the Examples. The second part will be in more general language.

For predicting impairments, the analysis of Papagno's data turned out to be not very illuminating, in that of all the possible patterns, only two (I and FI) would be inconsistent with the model; that is, the end result has been to emphasise the variety of impairment patterns in this dataset, which have led to a model with three systems. Regarding patients, the model suggests that if we want to identify groups that have damage to one system only (in order to then look for common features in their anatomy or physiology, for example), patients with impairment patterns $\mathrm{T}$ and TI form one group, those with impairment pattern $\mathrm{F}$ form another, and those with impairment patterns M and MI form a third. (And the remaining patients are more complex, in the sense of having damage to more than one system.) Turning now to the better understanding of what the tests are testing, the model suggests that a place to start is to ask what does the Idioms test have in common with the Token test, and what does it have in common with the Metaphors test. (The answer to this last question is that the Idioms and Metaphors tests are tests of figurative language.)

At the beginning of Section 3, it was said that double dissociation suggests, though it does not imply, the existence of different systems. A way in which double dissociation can result from a single change is if there is a nonmonotonic relation to performance, that is, increasing something either increases or decreases performance, depending on the starting point. A cross-over in performance does not then imply two systems. In the cognitive impairments context, Figure 1 of Kello (2003) shows the results of computer simulation of the "integrated pathway" 
model of word reading: changing one parameter improves performance on one task and impairs performance on another. My opinion is that a complicated model and a computer simulation are not needed: the essential thing is the nonmonotonic dependence of performance on a variable that is itself made up from a task characteristic and impairment. In psychology, a variable that is often said to have a nonmonotonic effect on performance is arousal: either too much or too little arousal impairs performance. In microbiology, it might be that each organism has a tolerance range of $\mathrm{pH}$; compound 1 lowers $\mathrm{pH}$, and the effect on $\mathrm{A}$ is to inactivate it by lowering the $\mathrm{pH}$ below the tolerance range while the effect on $\mathrm{B}$ is to leave it within its own tolerance range; and compound 2 affects $\mathrm{pH}$ also, but inactivates $\mathrm{B}$ by raising the $\mathrm{pH}$ too much, while leaving $\mathrm{A}$ within its tolerance range. If a nonmonotonic relationship were considered plausible, the logic of double dissociation, including the derivations of different systems as in the present paper, would have to be discarded.

The examples in this paper have shown that step-by-step building up of two or three systems can succeed with a small dataset. Sometimes there is a unique solution, sometimes several solutions are consistent with the data, and sometimes there is no solution. But, as far as I know, exhaustive consideration of all possibilities (by computer) is the only way of guaranteeing that the best theory is found. This was practicable with Example 2 (Hutchinson, 2003), but to find a good model for a large dataset might require more sophisticated processing. Speculation about details of method, whether for considering all possibilities or of some other strategy, is beyond this paper. Very likely, details would depend on the features built in to the software used, the size of the dataset, and on the exact aim (finding the best model, possibly not fully consistent with the dataset, might require quite a different method from finding a model that is fully consistent).

The strategy of research here is to adopt an initial aim of deducing a twosystem or three-system model directly from the data. This comes before discussing previous work, or making comparisons with theories, or considering qualitative differences between the columns of the dataset (e.g., tests and what they appear to be testing, or dermatophytes and their biological characteristics) or between the rows (e.g., the patients and their lesions, or compounds and their chemical properties). Consequently, as no theory has been used to generate the model, comparison with preexisting theories is not biased. In the case of cognitive impairments, no lesion data was used, and thus there would not be bias when searching for common features of the lesions in patients with damage to a particular system (inferred from the pattern of impairments); in the case of Example 3 , no data on the chemical and physical properties of the compounds was used, and thus there would not be bias when searching for relationships between these properties and the strengths of the compounds in inactivating the hypothesised 
two systems.

As was conceded in the Introduction, the systems that are hypothesised on the basis of observed patterns may be illusory. A statistician may process a table of 0's and 1's in an exploratory spirit, but then an expert in the subject matter of the table may reject the process or the results because they do not fit with the science. My view is that for patterns of cognitive impairments, the method of processing the patterns follows naturally from the conventional interpretation of double dissociation. But whether the method is appropriate for other areas of application (Section 5) is an open question.

The proposed method of processing data and expressing conclusions achieves two worthwhile goals. First, the results from the table of 0's and 1's alone are shown, uncontaminated by theories or previous empirical work or knowledge of how the rows differ from each other or how the columns differ from each other. Second, the presentation to the reader is briefer and clearer than a conventional narrative account.

\section{Acknowledgements}

The Centre for Automotive Safety Research, University of Adelaide, receives core funding from the Motor Accident Commission (South Australia) and the Department for Transport, Energy and Infrastructure (South Australia). Views expressed in this paper are those of the author, and do not necessarily represent those of the University of Adelaide or the sponsoring organisations.

\section{References}

Beltrametti, F., Lazzarini, A., Brunati, C., Marazzi, A., Jovetic, S., Selva, E. and Marinelli, F. (2003). Production and characterization of monochlorinated and dechlorinated A40926 derivatives. Journal of Antibiotics 56, 773-782.

Coltheart, M. (2001). Assumptions and methods in cognitive neuropsychology. In The Handbook of Cognitive Neuropsychology: What Deficits Reveal About the Human Mind (Edited by B. Rapp), 3-21. Psychology Press.

Hutchinson, T. P. (2003). A notation for the structure of cognitive processes, with application to patterns of verb-processing impairment. Language and Cognitive Processes 18, 165-174.

Hutchinson, T. P. (2004). Analysis of datasets showing which compounds kill which organisms: Inferring two systems. European Journal of Medicinal Chemistry 39, 107-111.

Hutchinson, T. P. (2005). Patterns of melanoma antigen-A expression in lung cancer patients. Chest, to appear as a Letter. 
Kello, C. T. (2003). The emergence of a double dissociation in the modulation of a single control parameter in a nonlinear dynamical system. Cortex 39, 132-134.

Kemmerer, D., Tranel, D. and Barrash, J. (2001). Patterns of dissociation in the processing of verb meanings in brain-damaged subjects. Language and Cognitive Processes 16, 1-34. (Addendum: 16, 461-463.)

Matysiak, J., Niewiadomy, A., Macik-Niewiadomy, G. and Korniłłowicz, T. (2000). Dependence of fungistatic activity of 2,4-dihydroxythiobenzanilides on the structure and lipophilic nature of the compounds. European Journal of Medicinal Chemistry 35, 393-404.

McCloskey, M. (2003). Beyond task dissociation logic: A richer conception of cognitive neuropsychology. Cortex 39, 196-202.

Mecklenberg, I., Stratakis, D. F., Huber, R. M., Haussinger, K., Morresi-Hauf, A., Riethmuller, G. and Kufer, P. (2004). Detection of melanoma antigen-A expression in sputum and bronchial lavage fluid of patients with lung cancer. Chest $\mathbf{1 2 5}(5$, Supplement), 164S-166S.

Papagno, C. (2001). Comprehension of metaphors and idioms in patients with Alzheimer's disease. A longitudinal study. Brain 124, 1450-1460.

Singh, M. P., Kong, M., Janso, J. E., Arias, D. A., Suarez, P. A., Bernan, V. S., Petersen, P. J., Weiss, W. J., Carter, G. and Greenstein, M. (2003). Novel $\alpha$ pyrones produced by a marine Pseudomonas sp. F92S91: Taxonomy and biological activities. Journal of Antibiotics 56, 1033-1044.

Vastag, M., Papp, T., Kasza, Z. and Vágvölgyi, C. (1998). Differentiation of Rhizomucor species by carbon source utilization and isoenzyme analysis. Journal of Clinical Microbiology 36, 2153-2156.

Yen, W. M. (1992). Item response theory. In Encyclopedia of Educational Research, 6th Edition, Volume 2 (Edited by M. C. Alkin), 657-667. Macmillan.

Received April 27, 2005; accepted May 22, 2006.

T. P. Hutchinson

Centre for Automotive Safety Research

University of Adelaide

South Australia 5005

Australia

paul@casr.adelaide.edu.au 